\title{
Thin Polymer Layers with Superparamagnetic Properties
}

\author{
Kristin Trommer, Carina Petzold, and Bernd Morgenstern \\ Research Institute of Leather and Plastic Sheeting (FILK), 09599 Freiberg, Germany \\ Correspondence should be addressed to Kristin Trommer; kristin.trommer@filkfreiberg.de
}

Received 8 May 2015; Revised 31 August 2015; Accepted 1 September 2015

Academic Editor: Shin-ichi Yusa

Copyright (C) 2015 Kristin Trommer et al. This is an open access article distributed under the Creative Commons Attribution License, which permits unrestricted use, distribution, and reproduction in any medium, provided the original work is properly cited.

\begin{abstract}
Superparamagnetic particles were widely used in medical applications as well as for magnetic sensors and actuators. Generally, the size of the particles is in the range of $10-20 \mathrm{~nm}$. To use such particles in large-scale applications, a simple processing as well as the use of commercially available particles is required. Therefore superparamagnetic nanoparticles available on the market were incorporated in flexible polymer films and the magnetic properties of the films were investigated. At ambient temperature no significant hysteresis was observed, indicating the superparamagnetic properties. Films containing up to $25 \%$ nanoparticles were prepared. The films show a saturation magnetization of $13.8 \mathrm{Am}^{2} / \mathrm{kg}$ and a coercivity of 7 Oe at ambient temperature.
\end{abstract}

\section{Introduction}

The magnetic properties of ferromagnetic as well as ferrimagnetic materials show size-related effects [1-4]. In such materials the spin moments of the magnetic centres do not compensate completely; there occur a coupling of the magnetic moments in small areas (Weiss' domains). The dimensions of these domains are at a range between ten nanometres and some micrometres. Because of a random distribution concerning the orientation of Weiss' domains the bulk material seems to be nonmagnetic. An external magnetic field causes orientation of the domains in one direction. This orientation remains also after removing the external field and a permanent magnetization is preserved. If such materials are present in the form of separated nanoparticles which are single-domain, they show superparamagnetic properties. This means that these nanoparticles are oriented in an external magnetic field, whereas they are nonoriented without external magnetic field. In contrary to larger particles they show no remanence. To achieve this superparamagnetic effect, interaction between Weiss' domains must be prevented. Therefore usual methods are embedding the nanoparticles into a matrix or into coreshell-particles. Concerning the last option the magnetic nanoparticles can be both in the core and in the shell of the mostly spherical structures $[5,6]$. The latter is applied in medical technology where such structures are placed at certain parts of the body using an external magnetic field. They can act as Drug-Delivery-System [7], can act as contrast medium for MRI (magnetic resonance imaging) [8], or can be heated through an alternating current magnetic field [911]. Up to now, in the medical area the effects of the magnetic nanoparticles were studied most intensely and are already used for different applications.

Great potential is assigned to the superparamagnetic polymers for the application in sensors or actuators $[12,13]$. Contrary to the medical applications these are high-volume technical applications.

Previous studies are mostly based on a special synthesis of nanoparticles. Usually an in situ preparation and immediate incorporation of the particles tailored to the polymer system is carried out. As an example, the synthesis of magnetite particles of iron (III) chloride for the incorporation into silicone elastomers was considered by Evans et al. [13]. Especially for the PVC polymer system the only described method is the preparation and incorporation of a ferrofluid with DOP (dioctyl phthalate) as the carrier liquid was described by Yáñez-Flores et al. [14] and Rodríguez-Fernández et al. [15]. Using the ferrofluid, superparamagnetic properties were achieved. In contrast, the preparation of PVC-based nanocomposites using magnetic nanoparticles (CoNi-based) leads to ferromagnetic materials with a strong hysteresis 
$\left(\mathrm{Hc}(0 \mathrm{~K})=850 \mathrm{Oe}, \mathrm{Hc}(300 \mathrm{~K}) \geq 300 \mathrm{Oe}, T_{B}=630 \mathrm{~K}\right)$. These nanocomposites were first described in 2004 by Vázquez et al. [16]. Due to the large volume applications, for the production of polymer materials with superparamagnetic properties, commercially available magnetite nanoparticles should be incorporated into flexible polymer matrixes in a way that superparamagnetic character is achieved. For this purpose, the particle agglomerates were split and incorporated into liquid or paste-like polymer masses. After solidification of the masses the magnetic particles are embedded in the polymer matrix as separated particles. As the nanoparticles are technical products the primary particles show a certain batch-related particle size distribution. The investigations are focussed on the procedural possibilities of particle treatment as well as the magnetic properties to be achieved this way. For a classification of the results the particles or particle arrangements were visualized in the polymer layers with the help of SEM and AFM.

\section{Experimental}

2.1. Materials. The PVC-sheets were prepared by E-PVC EP7060 (Vinnolit GmbH \& Co. KG) and Hexamoll DINCH (BASF) as plasticizer. As magnetic nanoparticles Fe(II, III) oxide powder (magnetite), showing a spherical particle shape in the range of $20-30 \mathrm{~nm}, 98 \%$ were chosen.

The silicone sheets were prepared using a dimethylpolysiloxane of an additional curing LR-type (Wacker AG).

2.2. Preparation of Polymer Sheets Containing Nanoparticles. Every particle content is given in weight\%.

2.2.1. Preparation of a PVC-Solution. For processing the particles with a PVC-solution a $10 \%$ PVC-solution was prepared in cyclohexanone. In a temperature-controlled vessel the PVC-powder was added to the solvent under stirring. The mixture was further stirred for 3 hours at $30^{\circ} \mathrm{C}$ and $1,000 \mathrm{r} / \mathrm{min}$. Afterwards the PVC was completely dissolved. This solution was used as stock solution from which the quantities needed for the single tests were taken, respectively.

To incorporate the nanoparticles in the PVC-solution, the plasticizer and the magnetic nanoparticles were combined and dispersed homogeneously. A typical PVC-formulation contained $100 \mathrm{phr}$ of PVC and $50 \mathrm{phr}$ of DINCH. Relating to the overall formulation $10-25 \%$ of magnetite was used. The use of a special disperser additive was not necessary. After dispersion, the mass was further treated at a three-roll mill or a ball mill.

2.2.2. Preparation of a PVC-Plastisol. When using a PVCplastisol in a first step the magnetic particles in the plasticizer were processed at the three-roll mill or by means of a ball mill according to the following description. The additional formulation components PVC-powder and a defoamer were added to the resulting homogeneous mixture, stirred, and processed at the three-roll mill up to a gap of $10 \mu \mathrm{m}$. The mass for film preparation contained $100 \mathrm{phr}$ of PVC, $50 \mathrm{phr}$ of $\mathrm{DINCH}$, and $10 \%$ of magnetite relating to the overall formulation.
TABLE 1: Process conditions using the ball mill.

\begin{tabular}{lcc}
\hline Time $[\mathrm{min}]$ & Speed $[\mathrm{U} / \mathrm{min}]$ & Temperature $\left[{ }^{\circ} \mathrm{C}\right]$ \\
\hline 10 & 690 & 15 \\
10 & 930 & 14 \\
10 & 1140 & 13 \\
10 & 1320 & 14 \\
10 & 2550 & 20 \\
\hline
\end{tabular}

\subsubsection{Processing of Dispersions Containing Nanoparticles at the} Three-Roll Mill. The homogeneous dispersion was processed at a three-roll mill (120 EH-250 of EXAKT) in several passages. The roll mill consists of two roller gaps which can be operated with a defined roller gap or a defined force that presses the rolls together. After several process steps (gap mode $5 \mu \mathrm{m}$ followed by force mode $5 \mathrm{~N}, 15 \mathrm{~N}$, and $40 \mathrm{~N}$ ) parts of the mass were separated for characterization and further processing into PVC-sheets.

2.2.4. Processing of Dispersions Containing Nanoparticles Using a Ball Mill. For processing with the ball mill a temperature-controlled vessel was charged with $230 \mathrm{~mL}$ grinding balls and $230 \mathrm{~mL}$ grinding material. The grinding process was performed on a Dispermat with a grinding disc having two plates carried out according to the steps shown in Table 1 .

2.2.5. Preparation of Silicone Dispersions. Preparing silicone dispersions, the nanoparticle agglomerates were added to the liquid silicone and stirred for 10 minutes followed by processing at the three-roll mill using the gap mode. The mass was treated in several passages to a roller gap of $5 \mu \mathrm{m}$. Dispersions containing $10 \%$ and $30 \%$ nanoparticles were prepared.

2.2.6. Sheet Preparation. Films of the prepared PVC-masses as well as the silicone dispersions were prepared by blade coating. Therefore the compounds were spread out on release paper at a Mathis Labcoater in order to form thin layers which were dried for 3 minutes at $100^{\circ} \mathrm{C}$ and cured for 3 minutes at $160^{\circ} \mathrm{C}$ in case of PVC and for 4 minutes at $180^{\circ} \mathrm{C}$ in case of silicone. Afterwards the cured layers were delaminated from the release paper to become a thin film of $100 \mu \mathrm{m}$. To obtain defect-free films, the sheets were prepared by application of several individual coating layers.

\subsection{Characterization Methods}

2.3.1. SEM-Investigations. A QUANTA FEG 250 (FEI Company) scanning electron microscope was used to observe the particle alignment of the magnetic particles. All samples were sputtered with gold.

2.3.2. AFM-Measurements. An atomic force microscope of the type Nanowizard 3 of the company JPK Instruments was used for the structure measurements and the magnetic measurements (MFM) in the nanometre range. The scanning 
unit of the apparatus is able to detect a grid of up to $100 \times$ $100 \times 15$ microns ( $X Y Z$ direction).

The topographical images were obtained in tapping mode. The MFM-images were obtained in the hover mode; that is, on the first scan (trace), the cantilever directly scans the surface of the sample, and, on the second scan (hover mode retrace), the cantilever is raised to a user-defined height and follows the topographical pattern from the previous trace. On the first trace, short range interactions (i.e., van der Waals forces) have the most significant effect and the topography is imaged. On the hover mode retrace, long range interactions such as magnetic forces are most prevalent, and the MFM-image will therefore reflect the magnetic properties of the sample $[17,18]$. The measurements were performed with cantilevers of the type NSC18/Co-Cr/Al BS. These cantilevers provide high stability in tapping mode as well as high sensitivity to magnetic forces. The coating consists of a Co layer of about $60 \mathrm{~nm}$ thickness on the tip and backside of the cantilever. The Co layer is formed as a polycrystalline film, which allows steady permanent magnetization in the direction of the tip axis. The length of the cantilever is $225 \mu \mathrm{m}$ and the tip radius is $<60 \mathrm{~nm}$. For the evaluation the images of height, amplitude, and phase were used.

2.3.3. Magnetization Measurements. The magnetic properties were determined using a Superconducting Quantum Interference Device (VSM-SQUID) magnetometer. Measuring the magnetic properties the temperature dependence of the magnetization was measured at $300 \mathrm{~K}$ and $2 \mathrm{~K}$. The temperature dependence on the magnetization was determined in field cooled- (FC-) as well as zero field cooled- (ZFC-) experiments.

Analysing the FC- and ZFC-measurements the blocking temperature of magnetic nanostructure ensembles can be determined. The sample is cooled down without application of a magnetic field below the expected blocking temperature, usually to some Kelvins (ZFC). Thus, the magnetic moments of the individual nanostructures are "frozen" statistically and the magnetization of the sample is zero on average at $T=$ $0 \mathrm{~K}$. If now an external magnetic field is applied and the measuring temperature is increased, magnetization of the sample rises up to a maximum, which lies at the blocking temperature. At the maximum the thermal energy is sufficient to orientate all magnetic moments in the direction of the external field. A size distribution of the nanostructures leads to an expansion of the continuous increase. If the temperature rises above the blocking temperature, the magnetization of the nanostructures decreases according to Curie's law for a typical paramagnetic material with $1 / T$ (Figure 1).

In the $\mathrm{FC}$-experiment the sample is cooled in an external magnetic field and the magnetization is measured in this connection. For all measuring temperatures $T>T_{B}$ applies a $1 / T$-behaviour for the magnetization of the sample; for $T<T_{B}$ the magnetization further increases until saturation is reached. This occurs if all magnetic moments of the individual nanostructures are oriented through the external field.

Concerning single-domain nanostructures the thermal stability of the magnetization of a magnetic particle has a big influence on the total magnetization of the sample. For

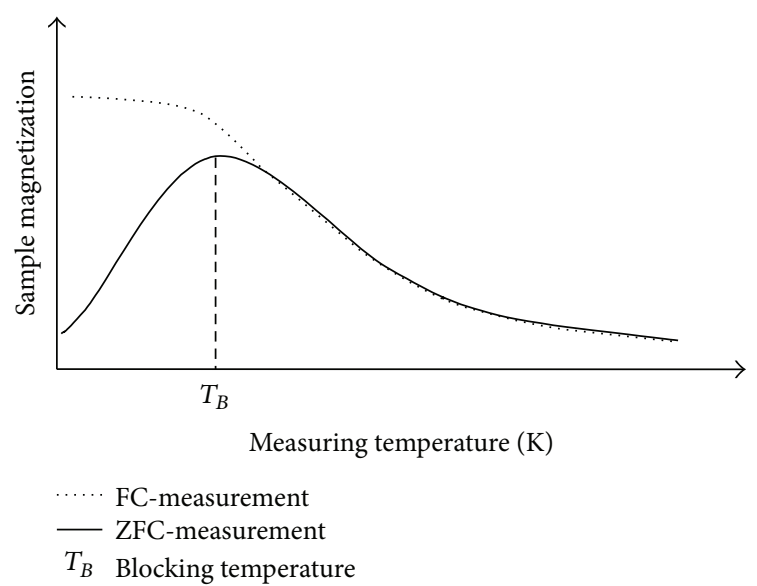

FIGURE 1: Scheme of the temperature-dependent magnetization in an FC- or ZFC-experiment.

an ensemble of particles with uniaxial anisotropy, which are oriented parallel to the axis of the magnetization in a generated magnetic field $B$, in case of relaxation, an energy barrier must be overcome (Figure 2(a)). The minimum energy, which is necessary for this purpose, can be described with the blocking temperature $\left(T_{B}\right)$. Above this temperature each particle reaches the thermal balance during the measuring time. This means that the magnetization of the particles within the measuring time jumps several times between the states parallel and antiparallel to the external field. As a consequence the hysteresis curve does not show remanence and coercivity. At temperatures below the blocking temperature the magnetic moment of a particle is blocked; that is, thermal energy is not sufficient anymore to overcome the potential peak. The magnetic moments can follow the external field only delayed which results in a hysteresis curve (Figure 2(b)).

\section{Results and Discussion}

3.1. Visualization of the Nanostructures. In SEM-investigations the particles were detected visually in the films. The images show that the size and the number of the existing particle agglomerates decrease with increasing force in the treatment process. An increasing number of primary particles are detected which are spatially separated. The major difference between the films from the plastisol and the solution is in the structuring of the plastisol-based layers. In the sheets prepared from the paste the areas of the PVC-particles (dark) are clearly noticeable (Figure 3(b)). These are free from magnetite. The magnetite is located exclusively in the phase of the thresholds between these particles high in plasticizer (light areas). This also means that the concentration of the magnetite particles is locally much higher than in the homogeneous film resulting from the solution (Figure 3(a)). Both models were characterized concerning their magnetic properties (see Section 3.2).

To get a more detailed idea of how the particles are present in the films, SEM- and AFM-images were made at microtome sections of the films. These investigations show that there 


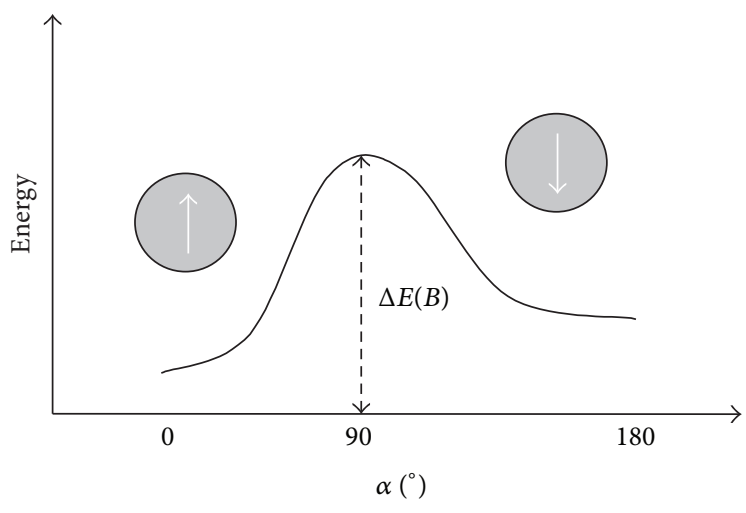

(a)

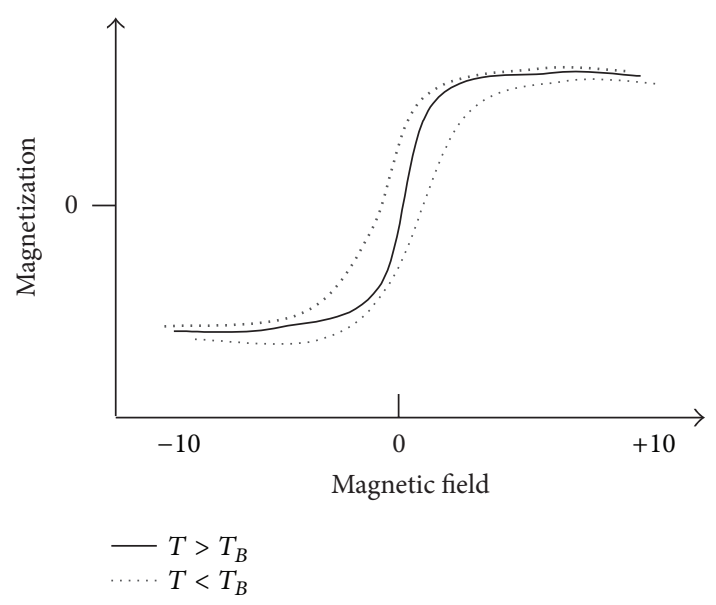

(b)

FIGURE 2: (a) Scheme of possible magnetization directions of a ferromagnetic particle in an external magnetic field $B$; $\alpha$ is the orientation of the magnetic moments in relation to the external magnetic field. (b) Typical magnetization curves above and below the blocking temperature.

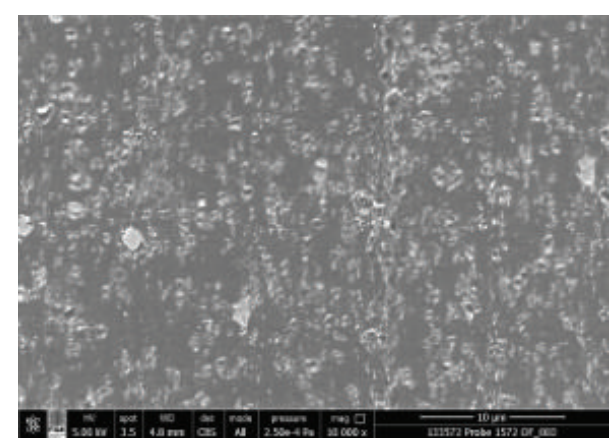

(a)

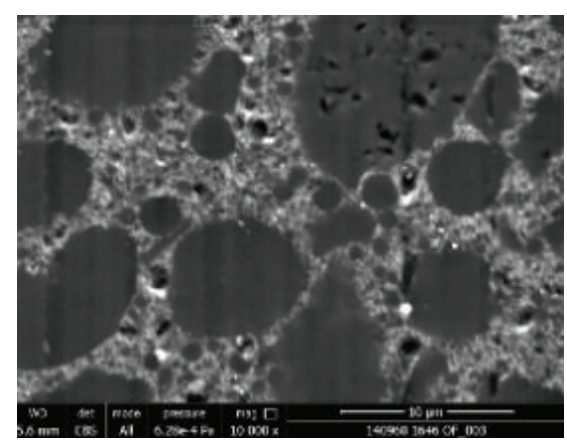

(b)

FIGURE 3: SEM-images of PVC-sheets prepared from solution (a) and the paste (b) at a magnification of 10,000.

are areas in which the particles exist separated. Obviously, the separation of the agglomerates into primary particles has been successful there. This can be seen in the detailed images of the SEM- and AFM-investigations (Figure 4 figures bottom). The resolution in the SEM is not sufficient to notice separate particles. In addition there is the effect that due to the massive element iron the electrons are gathered not only from the surface, but also from the deeper layers. It can be looked into the sample and thereby deeper lying particles are indistinguishable from those on the surface. In contrast to that the phase pictures of an AFM-image can make these particles very well visible. During the AFMmeasurement the cantilever swings with a certain frequency. Due to the interactions between sample surface and peak there is a phase shift between the stimulation frequency and the measured cantilever movement. This phase shift is shown as phase picture and thus gives information about the mechanical properties of the sample. Differences in the materials (hard/soft), which look the same in the height image, can be distinguished by light/dark areas. The light areas represent the hard magnetite particles and the darker ones the soft PVC-matrix. The picture below clearly shows the separation of the particles. The size of the particles is in the two-digit nanometre range $(50-60 \mathrm{~nm})$. Taking into consideration the measuring error which results from the peak radius, the values nearly correspond to the primary particles size of the magnetite of $20-30 \mathrm{~nm}$. According to analogue investigations of cut agglomerates the primary particles can also be seen in the agglomerates as individual particles.

For a clear statement about the magnetic conditions at the surface the phase pictures of an AFM-measurement (Figure 5(a)) with MFM-images of the same area (Figure 5(b)) are compared. It shows a film produced from a PVC-solution.

The MFM-images were taken with a hover height of $30 \mathrm{~nm}$ and show clear phase shifts. The phase shifts visible as dark areas do not correlate with the height signal and can thus be clearly assigned to the magnetic signal. A comparison with the distribution of the magnetite particles, which can be seen from the phase picture, confirms this. This proves the magnetization on the nanometre scale.

3.2. Magnetic Properties. The investigations concerning magnetization of the PVC-films depending on the external magnetic field revealed that the films produced both from 


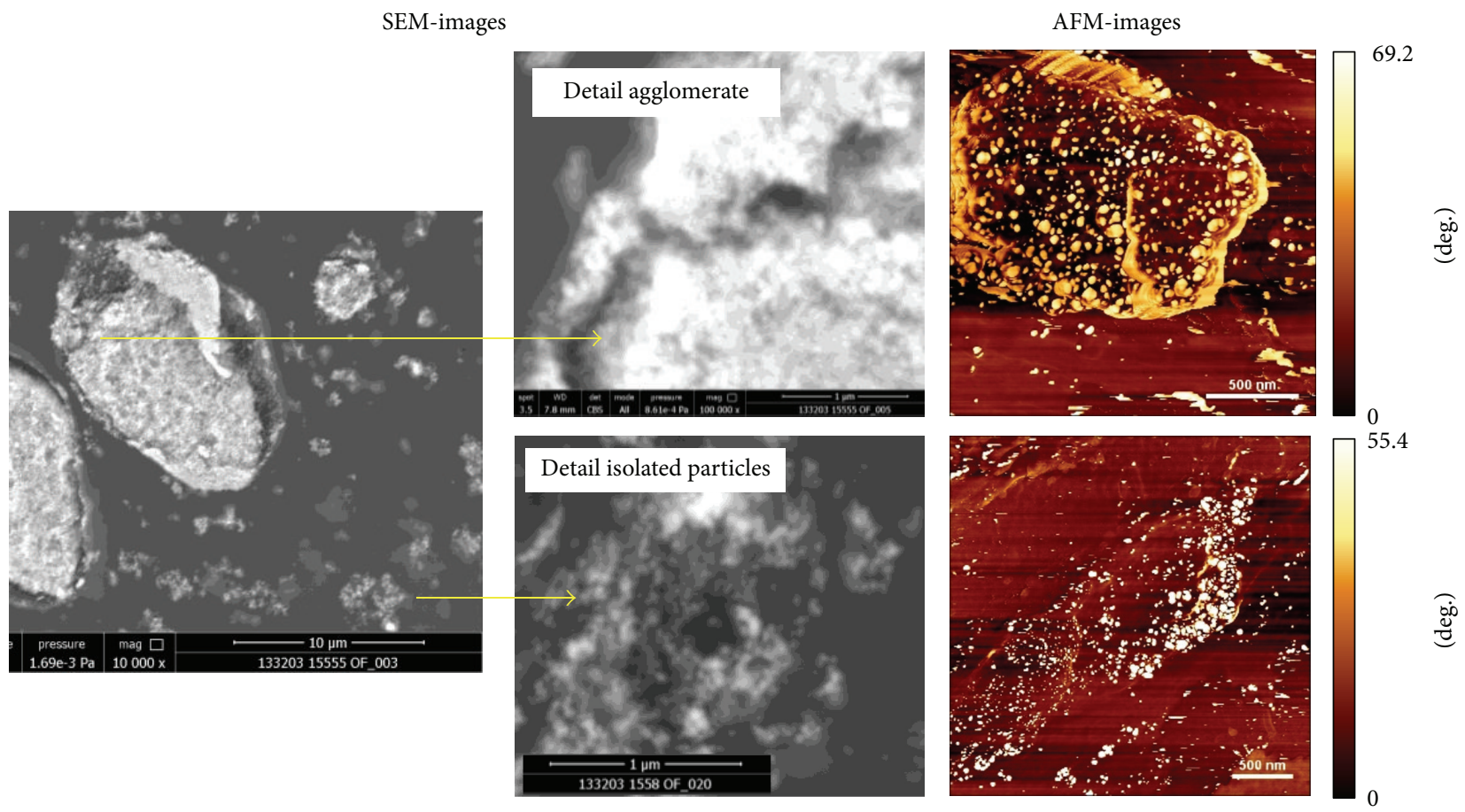

FIGURE 4: SEM- and AFM-images of a PVC-sheet prepared from a solution (microtome section); overview and detailed views showing the agglomerate (top) as well as isolated particles (bottom).

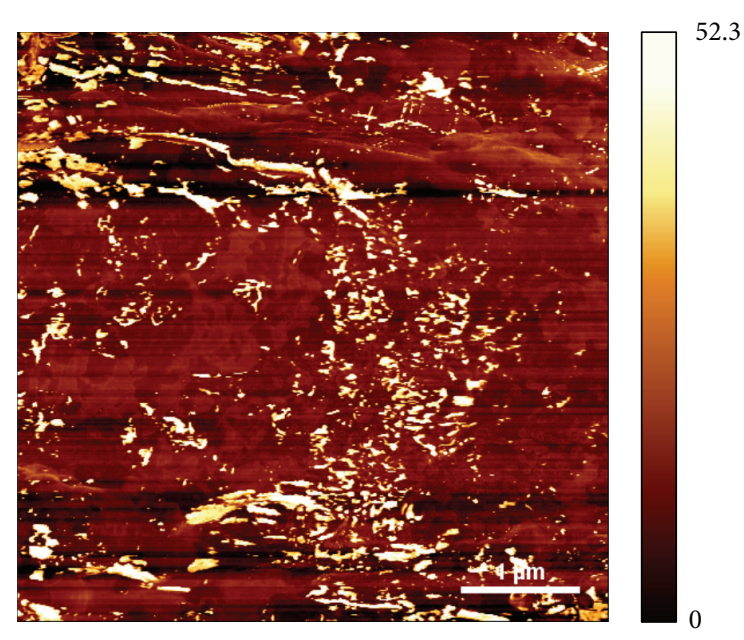

(a)

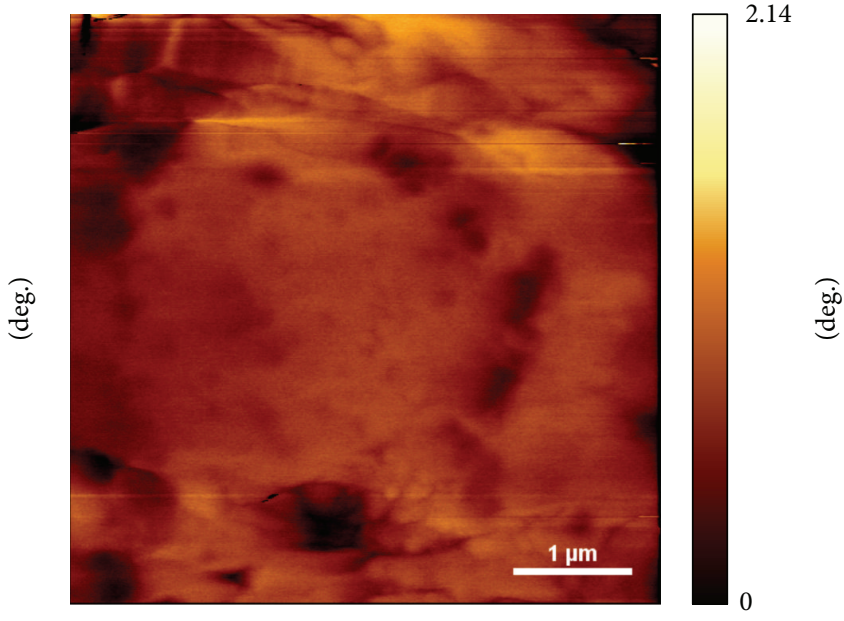

(b)

FIGURE 5: (a) AFM-image PVC-film from the solution; phase picture (trace); (b) MFM-images phase picture (retrace).

the solution and from the paste have superparamagnetic behaviour. The left side of Figure 6 shows an overview spectrum of films with $10 \%$ particle content. The hysteresis curves were taken above $(300 \mathrm{~K})$ and below $(2 \mathrm{~K})$ the blocking temperature $\left(T_{B}\right)$. In the top right side of Figure 6 the magnetization curves at $300 \mathrm{~K}$ in the area around the zeropoint are given. All curves show a very low remanence $\left(M_{\text {rem }}\right)$ of $<0.11 \mathrm{Am}^{2} / \mathrm{kg}$ and coercivity $\left(B_{C}\right)$ of 6 Oe. Investigations showed that small differences (up to $11 \mathrm{Oe}$ ) in the coercivity between the films cannot be attributed to the processing but to different particle batches. Against the background of a large-scale application in films, particles were used, which are available at a larger scale and reasonably priced. Since there are technical products, the particle size and the particle size distribution of the individual batches vary within certain limits. As expected during measurements below the blocking temperature of $2 \mathrm{~K}$ there is a hysteresis (Figure 6 bottom), as the magnetic moments can follow the change of the external field only delayed. At $2 \mathrm{~K}$ the measuring values for the remanence reach 1.9-2.1 $\mathrm{Am}^{2} / \mathrm{kg}$ and the coercivity of 275 Oe.

The method of particle treatment does not have an influence on the coercivity, but on the resulting saturation 


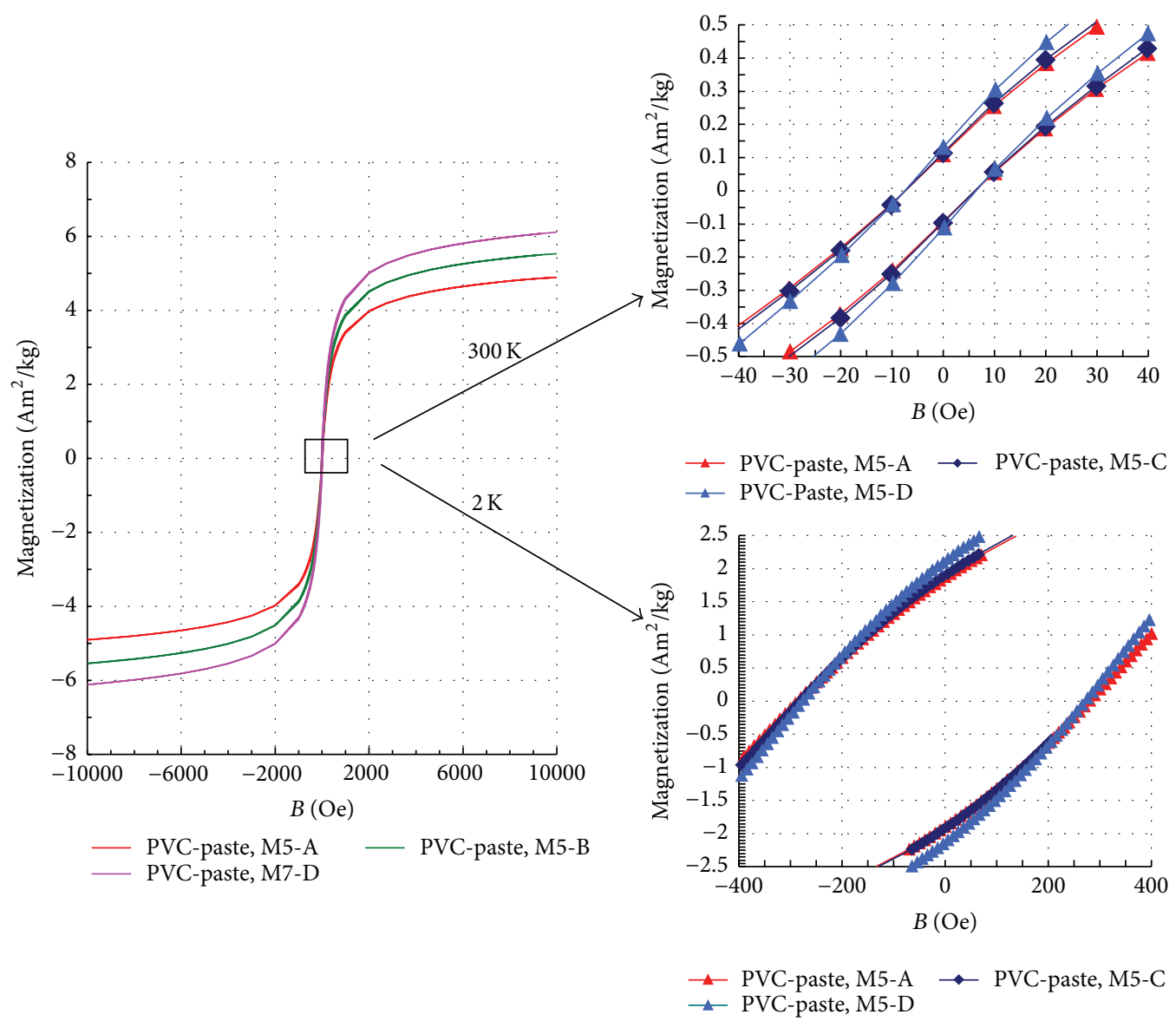

FIGURE 6: Left: magnetization curves of sheets containing 10\% nanoparticles prepared from PVC-pastes using a three-roll mill; the force in the gap was increased from A via B to D. Top right: hysteresis at $300 \mathrm{~K}$, bottom right: hysteresis at $2 \mathrm{~K}$.

magnetization. Films produced of masses which were treated with small force show a low saturation magnetization of $4.9 \mathrm{Am}^{2} / \mathrm{kg}$. Compared to this, films produced of masses which were treated with the highest possible force show a saturation magnetization of $6.1 \mathrm{Am}^{2} / \mathrm{kg}$, which is about $24 \%$ higher.

A dependence of the remanence on the process conditions was not found. The remanence for films, the paste of which was treated at the three-roll mill, is between $0.08 \mathrm{Am}^{2} / \mathrm{kg}$ and $0.11 \mathrm{Am}^{2} / \mathrm{kg}$. For a practical use this means that after switching off an external magnetic field the films do not show a permanent magnetization.

The processing in the paste does not allow a higher particle content than $10 \%$, whereas preparation in the solution allows the incorporation of much more particles. Thus, also the resulting saturation magnetization increases. Figure 7 shows the hysteresis curves of PVC-films containing $10 \%$, $15 \%$, and $25 \%$ nanoparticles. The highest reachable saturation magnetization is at $13.8 \mathrm{Am}^{2} / \mathrm{kg}$ for a particle content of $25 \%$. The curves in the area of the zero-point (Figure 7(a)) show an increase of the remanence from $0.121 \mathrm{Am}^{2} / \mathrm{kg}(10 \%)$ to $0.207 \mathrm{Am}^{2} / \mathrm{kg}(15 \%)$ and $0.368(25 \%)$ whereas the coercivity is unchanged for all samples at $7 \mathrm{Oe}$.
Using FC- and ZFC-experiments the blocking temperatures of the magnetic nanostructure ensembles were determined. For an ideal superparamagnet the ZFC-curve has a maximum at the blocking temperature; above the blocking temperature the curves for FCM and ZFCM coincide. Figure 8(b) shows the course of the curves for the PVC-films containing $10 \%, 15 \%$, and $25 \%$ nanoparticles $(20-30 \mathrm{~nm})$ as well as with a content of $10 \%$ larger primary particles.

The significant difference in the magnetic behaviour also becomes apparent in this experiment. The ZFC-curve of the PVC-film, which was produced with larger nanoparticles, does not have a maximum and does not converge with the FC-curve up to a value of $350 \mathrm{~K}$; for example, it shows ferrimagnetic behaviour in this area. The PVC-films with smaller nanoparticles show a maximum in the ZFC-curve between $150 \mathrm{~K}$ and $180 \mathrm{~K}$ and converge in the area between $250 \mathrm{~K}$ and $300 \mathrm{~K}$. The exact temperatures are different for the different preparation processes. Using the example of films produced from pastes this is illustrated in Figure 8(a). For a better overview the ZFC-curves are shown only. From the curves the blocking temperatures (Table 2) were assessed. The values show a clear tendency; the higher the application of force in the gap is the further the blocking temperature 

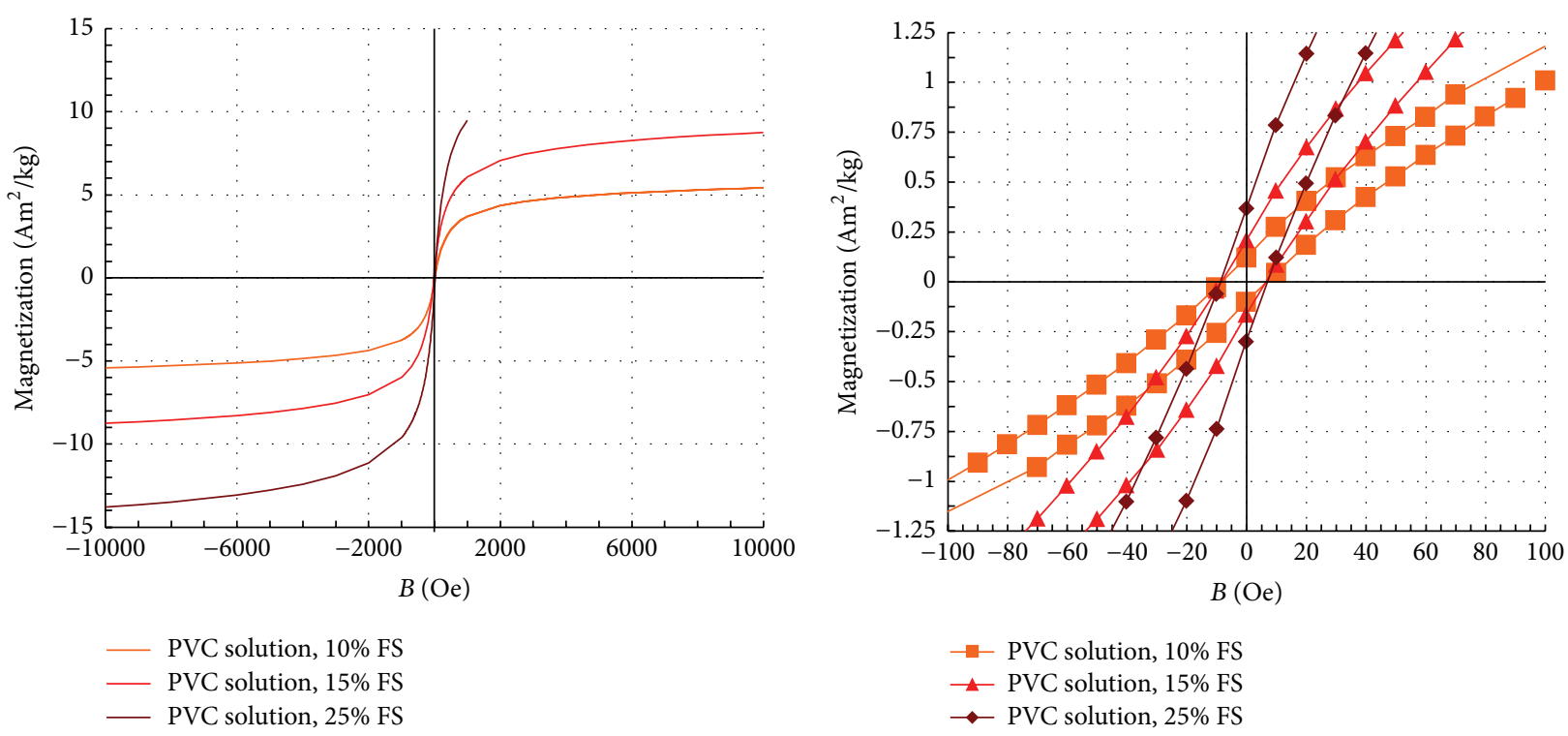

(a)

(b)

FIGURE 7: Magnetization curves of sheets containing 10\%, 15\%, and 25\% nanoparticles prepared from PVC-solution using a ball mill.

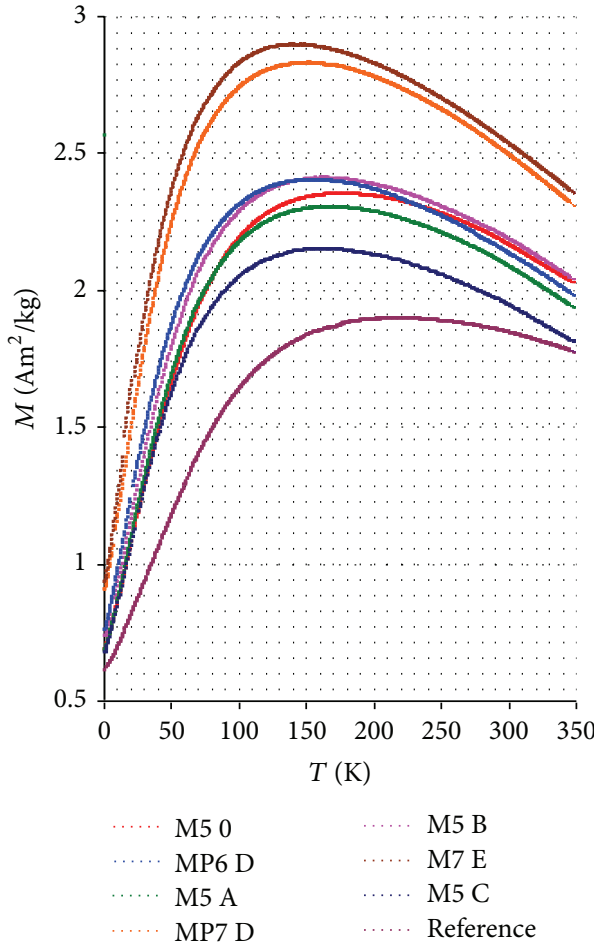

(a)

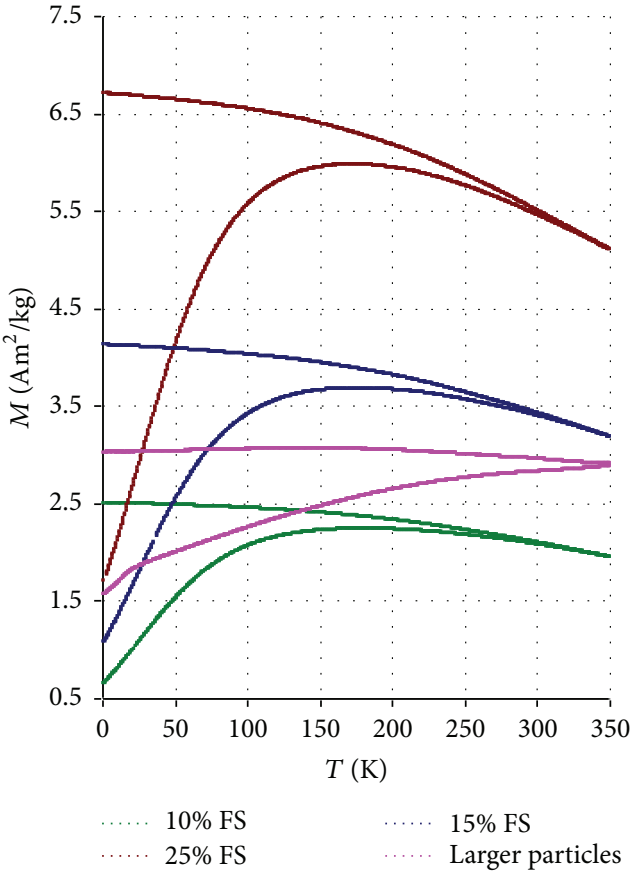

(b)

Figure 8: (a) Magnetization curves of sheets containing 10\% nanoparticles processed at different modes (process 0, A, B, and C); (b) magnetization curves of sheets containing 10\%, 15, and 25\% nanoparticles, prepared from PVC-solutions via ball mill.

is shifted to lower values. The difference of the blocking temperature is about $40 \mathrm{~K}$ between reference value and the easiest treatment in the gap mode with $10 \mu \mathrm{m}$ or $5 \mu \mathrm{m}$ gap setting. From this first treatment stage up to the most complex treatment with several passages in force mode with a force of
$20 \mathrm{~N}$ or $38 \mathrm{~N}$ the blocking temperatures differ by about $35 \mathrm{~K}$. As there are different nanostructure ensembles in the films the maximum of the ZFC-curve is wide and converges with the FC-curve after the maximum only. The more consistent the particles in their size are and the less interactions there 
TABLE 2: Blocking temperature in dependence of mass processing.

\begin{tabular}{lcl}
\hline $\begin{array}{l}\text { Sample } \\
\text { labeling }\end{array}$ & $\begin{array}{c}\text { Range of } \\
\text { curves-maximum } \\
T_{B}[\mathrm{~K}]\end{array}$ & Mass processing \\
\hline Reference & $205-235$ & Magnetite powder \\
MP5 0 & $170-185$ & Gap 1: 10 $\mu \mathrm{m}$; gap 2: $5 \mu \mathrm{m}$ \\
MP5 A & $162-174$ & Gap 1: 2 N; gap 2: 5N \\
MP5 B & $157-178$ & Gap 1: 5 N; gap 2: 15 N \\
MP5 C & $153-175$ & Gap 1: 10 N; gap 2: 30 N \\
MP6 D & $145-160$ & MP5 C + additional dispersion \\
MP7 D & $142-160$ & 3 passages gap 1: 11 N; gap 2: 33 N \\
MP7 E & $130-150$ & MP7 D + 3 passages gap 1: 20 N; gap \\
\hline
\end{tabular}

are between the particles or clusters the more the curve progression corresponds to that of an ideal superparamagnet.

The selected ferrite particles are suitable for the processing with different polymer systems. In order to show this, the particles were incorporated into liquid silicones at the three-roll mill using the calendering process. Silicone films were produced from the contained masses and their magnetic properties were analysed analogue to the PVC-films. The measured values for the remanence of about $0.09 \mathrm{Am}^{2} / \mathrm{kg}$ as well as the coercivity of about 10 Oe do not differ from the respective values of the PVC-films.

\section{Conclusions}

For the first time the preparation of PVC-films with superparamagnetic properties by incorporation of commercially available magnetite nanoparticles (particle sizes of 20-30 nm) was successful. The nanocomposites could be prepared using a ball mill as well as a three-roll mill for processing the particle agglomerates. Applying the three-roll mill it was found that the higher the application of a force in the roll gap the higher the saturation magnetization and the lower the blocking temperature. In general the blocking temperature of the films is below $185 \mathrm{~K}$, in case of optimum particle treatment below $150 \mathrm{~K}$. The processing parameters did not have an influence on the remanence and the coercivity. The reachable values for the remanence in samples containing $10 \%$ nanoparticles were at $0.11 \mathrm{Am}^{2} / \mathrm{kg}$ and those of the coercivity at 5,7 $\mathrm{Oe}(300 \mathrm{~K})$. For an external magnetic field of 70,000 Oe a maximum saturation magnetization of $6.1 \mathrm{Am}^{2} / \mathrm{kg}$ was reached for these composites. In case of processing from the solution higher particle contents can be realized. For films with 25\% particles the achievable saturation magnetization is at $13.8 \mathrm{Am}^{2} / \mathrm{kg}$, which is much higher than the described values using ferrofluids (maximum 2.0 $\mathrm{Am}^{2} / \mathrm{kg}$ ) [15].

In practice this means if the films are located in an external magnetic field, they behave like an ferromagnetic material. They are magnetized and move in the direction of the magnetic field, or they can be detected in the magnetic field. If the field strength reaches the value of 0 Oe (switching off the external field) the PVC-film is demagnetized again; that is, without external magnetic field the film shows the properties typical for plastics. As there is no permanent magnetization, ferrous metals are not influenced by the film. It follows that the films have no hysteresis and thus no remanence, that is, having superparamagnetic behaviour in use at room temperature.

The use of the magnetite nanoparticles in other polymer systems with similar mass preparation is possible. This was proven using the example of silicone films.

\section{Conflict of Interests}

The authors declare that there is no conflict of interests regarding the publication of this paper.

\section{Acknowledgments}

The research project "Modification of plasticized PVC with functional nanoparticles," Registration no. VF110026, was partially funded from budget funds of the Bundesministerium für Wirtschaft und Energie (BMWi-Federal Ministry of Economics and Technology). The authors are grateful for the support granted.

\section{References}

[1] H. Goesmann and C. Feldmann, "Nanoparticulate functional materials," Angewandte Chemie International Edition, vol. 49, no. 8, pp. 1362-1395, 2010.

[2] S. Behrens, "Preparation of functional magnetic nanocomposites and hybrid materials: recent progress and future directions," Nanoscale, vol. 3, no. 3, pp. 877-892, 2011.

[3] E. J. Choi, Y. Ahn, and E. J. Hahn, "Size dependence of the magnetic properties in superparamagnetic zinc-ferrite nanoparticles," Journal of the Korean Physical Society, vol. 53, no. 4, pp. 2090-2094, 2008.

[4] A.-H. Lu, E. L. Salabas, and F. Schüth, "Magnetic nanoparticles: synthesis, protection, functionalization, and application," Angewandte Chemie, vol. 46, no. 8, pp. 1222-1244, 2007.

[5] N. H. Hai, N. H. Luong, N. Chau, and N. Q. Tai, "Preparation of magnetic nanoparticles embedded in polystyrene microspheres," Journal of Physics: Conference Series, vol. 187, no. 1, 2009.

[6] O. Philippova, A. Barabanova, V. Molchanov, and A. Khokhlov, "Magnetic polymer beads: recent trends and developments in synthetic design and applications," European Polymer Journal, vol. 47, no. 4, pp. 542-559, 2011.

[7] Q. A. Pankhurst, J. Connolly, S. K. Jones, and J. Dobson, "Applications of magnetic nanoparticles in biomedicine," Journal of Physics D: Applied Physics, vol. 36, no. 13, pp. R167-R181, 2003.

[8] H. B. Na, I. C. Song, and T. Hyeon, "Inorganic nanoparticles for MRI contrast agents," Advanced Materials, vol. 21, no. 21, pp. 2133-2148, 2009.

[9] A. Jordan, P. Wust, H. Fählin, W. John, A. Hinz, and R. Felix, "Inductive heating of ferrimagnetic particles and magnetic fluids: physical evaluation of their potential for hyperthermia," International Journal of Hyperthermia, vol. 9, no. 1, pp. 51-68, 1993. 
[10] A. Jordan, K. Maier-Hauff, P. Wust, and M. Johannsen, "Nanoparticles for thermotherapy," in Nanoparticles for the Life Sciences, Wiley-VCH, 2007.

[11] M. Johannsen, B. Thiesen, P. Wust, and A. Jordan, "Magnetic nanoparticle hyperthermia for prostate cancer," International Journal of Hyperthermia, vol. 26, no. 8, pp. 790-795, 2010.

[12] Z. Varga, J. Fehér, G. Filipcsei, and M. Zrínyi, "Smart nanocomposite polymer gels," Macromolecular Symposia, vol. 200, no. 1, pp. 93-100, 2003.

[13] B. A. Evans, B. L. Fiser, W. J. Prins et al., "A highly tunable silicone-based magnetic elastomer with nanoscale homogeneity," Journal of Magnetism and Magnetic Materials, vol. 324, no. 4, pp. 501-507, 2012.

[14] I. G. Yáñez-Flores, R. Betancourt-Galindo, J. A. M. Aquino, and O. Rodríguez-Fernández, "Preparation and characterization of magnetic PVC nanocomposites," Journal of Non-Crystalline Solids, vol. 353, no. 8-10, pp. 799-801, 2007.

[15] O. S. Rodríguez-Fernández, C. A. Rodríguez-Calzadíaz, I. G. Yáñez-Flores, and S. M. Montemayor, "Preparation and characterization of a magneto-polymeric nanocomposite: $\mathrm{Fe}_{3} \mathrm{O}_{4}$ nanoparticles in a grafted, cross-linked and plasticized poly(vinyl chloride) matrix," Journal of Magnetism and Magnetic Materials, vol. 320, no. 14, pp. e81-e84, 2008.

[16] M. Vázquez, C. Luna, M. P. Morales, R. Sanz, C. J. Serna, and C. Mijangos, "Magnetic nanoparticles: synthesis, ordering and properties," Physica B: Condensed Matter, vol. 354, no. 1-4, pp. 71-79, 2004.

[17] D. Sarid, Scanning Force Microscopy-With Applications to Electric, Magnetic and Atomic Forces, Oxford University Press, New York, NY, USA, 1994.

[18] A. Körnig, M. A. Hartmann, C. Teichert, P. Fratzl, and D. Faivre, "Magnetic force imaging of a chain of biogenic magnetite and Monte Carlo analysis of tip-particle interaction," Journal of Physics D: Applied Physics, vol. 47, no. 23, Article ID 235403, 2014. 

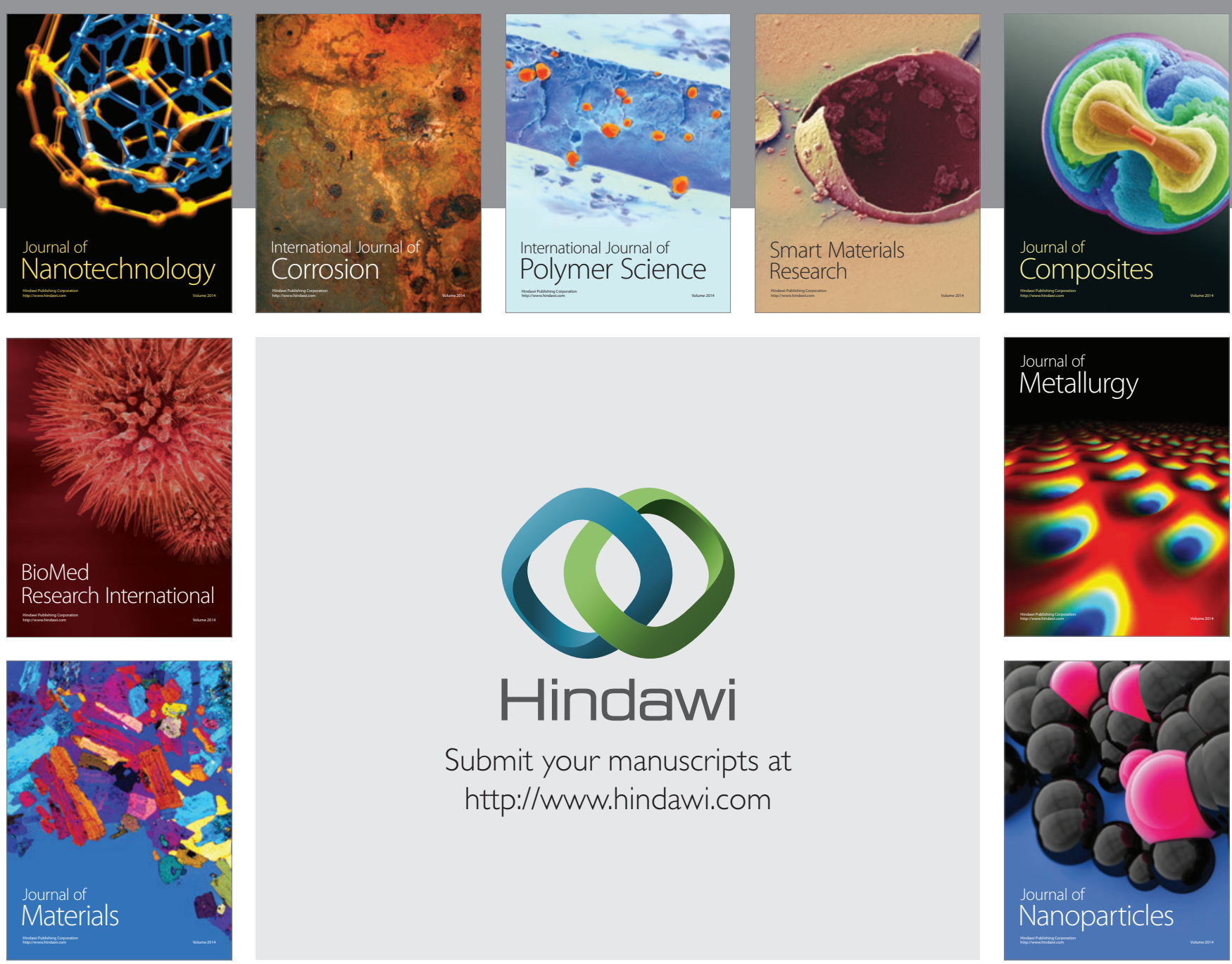

Submit your manuscripts at http://www.hindawi.com
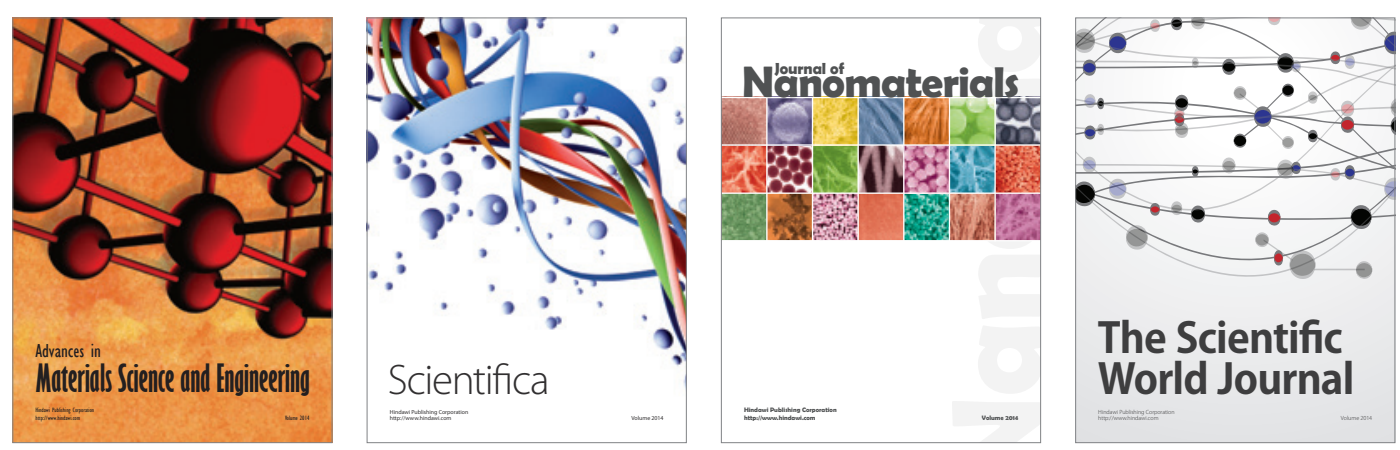

\section{The Scientific World Journal}
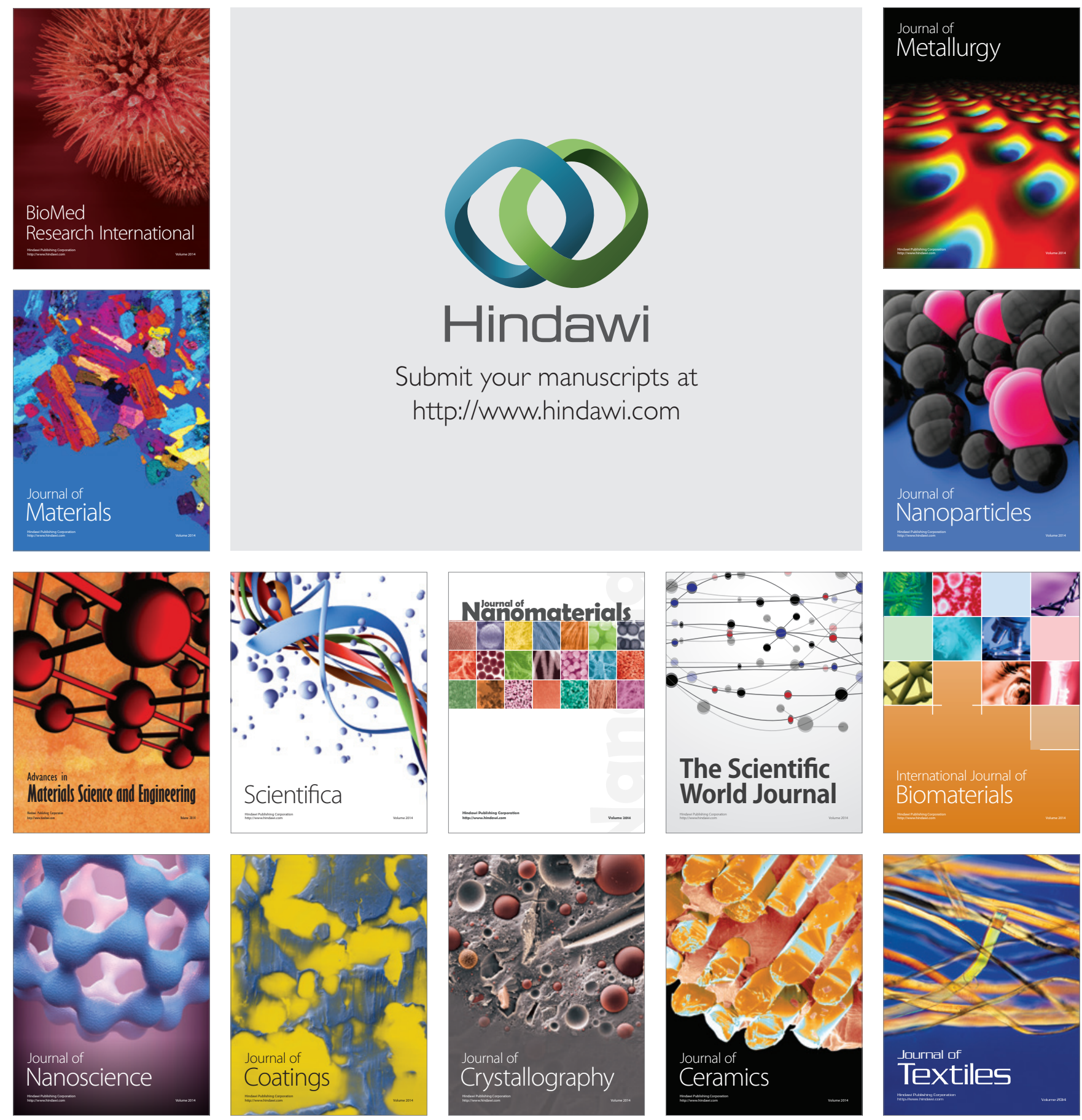